\title{
Resonance structures in elastic and Raman scattering from microspheres
}

\author{
Chak K. Chan, Richard C. Flagan, and John H. Seinfeld
}

\begin{abstract}
To study the interactions between Mie scattering and Raman emissions of spherical particles, we measured the Raman spectra together with the elastically scattered light of the excitation source of an evaporating aqueous sodium nitrate droplet. Resonance structures were observed in the temporal profiles of the elastically scattered light and Raman nitrate and water emissions. The resonance structures in these three profiles occurred in a concerted mode but sometimes occurred independently of each other. A model of inelastic scattering by microspheres by Kerker et al. ["Raman and Fluorescent Scattering by Molecules Embedded in Spheres with Radii up to Several Multiples of the Wavelength," Appl. Opt. 18, 1172-1179 (1979); "Lorenz-Mie Scattering by Spheres: Some Newly Recognized Phenomena," Aerosol Sci. Technol. 1, 275-291 (1982); "Inelastic Light Scattering," in Aerosol Microphysics I: Particle Interaction, W. H. Marlow, Ed. (Springer-Verlag, New York, 1980); "Model for Raman and Fluorescent Scattering by Molecules Embedded in Small Particles," Phys. Rev. A 13, 396-404 (1976)] and the behavior of low order Mie resonances were used to explain the data. This type of data can be used for the determination of chemical compositions of spherical particles.
\end{abstract}

\section{Introduction}

Spectroscopic techniques such as Raman spectroscopy can be used to determine the chemical composition of single suspended particles. ${ }^{1}$ Raman spectroscopy of aerosol particles was first applied by analyzing microparticles on a substrate. ${ }^{2}$ Recently, Raman spectroscopy has been applied to levitated single microparticles using either the electrodynamic balance or optical levitation. Preston et al..$^{3}$ measured the Raman spectra of optically levitated dioctyl phthalate droplets. Thurn and Kiefer also used optical levitation to study Raman emissions of glass microspheres ${ }^{4,5}$ and of water and glycerol droplets. ${ }^{6}$ Using an electrodynamic balance, Fung and Tang ${ }^{7-10}$ studied Raman emissions from droplets of aqueous solutions of various inorganic salts such as sulfates and nitrates. By using a high resolution monochromator, they observed fine spectral structures indicating phase transitions in supersaturated droplets and cation effects on the $\mathrm{Ra}$ man spectra of anions (e.g., sulfates and nitrates).

\footnotetext{
All authors are with Department of Chemical Engineering, California Institute of Technology, Pasadena, California 91125.

Received 22 February 1990.

0003-6935/91/040459-09\$05.00/0.

(C) 1991 Optical Society of America.
}

Although identification of the chemical nature of single suspended particles using Raman spectroscopy has been demonstrated, quantitative Raman analysis of spherical particles for chemical composition determination is complicated because of the coupling with Mie scattering. Benner et al. ${ }^{11}$ observed structural resonances in the fluorescence spectra from dye impregnated polystyrene microspheres suspended in water. Resonance structures in Raman spectra of levitated spherical particles have also been reported. Thurn et $a l .{ }^{6}$ observed resonance structures superimposed on the broad spectrum seen in bulk sample spectra in the $\mathrm{O}-\mathrm{H}$ stretching region of the Raman spectra of a 1:6 water-glycerol droplet mixture. They identified individual peaks of the measured Raman spectrum as Mie resonance peaks. Preston et al. ${ }^{3}$ reported similar resonance structures in the Raman spectra of the C-H stretching region of optically levitated dioctyl phthalate droplets. As the droplet evaporated due to heating from the argon laser used as the excitation source and also that for optical levitation, the resonance peaks in the Raman spectra shifted systematically with time. Moreover, the observed resonance structures in the measured Raman spectra changed when different droplet sizes were used.

The resonance peaks in inelastic spectra of spherical particles reported by the above mentioned researchers can be attributed to morphology dependent resonances that are strong functions of particle size and optical properties. Since a minute change in size that 
occurs during slow evaporation can result in large fluctuations in scattering intensity, this sensitivity of the scattered light intensity can be used to determine particle size to 1 part in $10^{5}$. The refractive index of the particle and the size parameter, which is the ratio of particle circumference to the optical wavelength of interest, determine the locations of these resonances. In Raman scattering, the wavelengths involved are the excitation laser wavelength and those of the Raman emissions. If any wavelength involved in the process corresponds to a resonant wavelength of the particle, the scattered intensity will be significantly affected by Mie resonances.

Raman emissions can be envisioned to occur as a result of a two-step process. The first is the excitation of the Raman active molecules by absorption of photons of incident frequency. The second step is the release of photons at a shifted frequency when the excited molecules fall back to their ground states. The local emitted Raman intensity is proportional to the local excitation field intensity. For a bulk sample, the local field strength is relatively uniform or can be corrected for the Gaussian nature of the excitation laser beam when the dimension of the scattering volume is comparable to or larger than the beam waist of the laser beam. On the other hand, in the case of Mie scattering, the local excitation field intensity inside the particle is strongly dependent on the size and refractive index of the particle. Hence Raman scattering from spherical particles can be complicated by Mie scattering in two ways. Since the internal excitation field drives the Raman emissions, one expects Raman emissions at all wavelengths to be enhanced when the incident excitation itself is at a Mie resonance. When the Raman shifted light corresponds to a resonant wavelength, the emission intensity at that particular wavelength will be selectively enhanced.

Understanding of the interactions of Mie scattering and Raman emissions is crucial to the application of quantitative Raman spectroscopy of spherical particles. Kerker and associates ${ }^{12-15}$ have modeled inelastic scattering from microspheres, predicting the intensities of the elastically scattered light and the Raman emissions as a function of particle size and optical properties. Although resonance peaks in Raman spectra have been reported by various researchers, ${ }^{3-8}$ systematic studies of resonance peaks in the elastically scattered light and Raman emissions as a function of the size parameter have not been presented. Such data are necessary for the validation of quantitative models of Raman scatterings from microspheres and the ultimate application of this technique to quantitative analysis.

We present results of some experimental studies relating to the interactions of resonances in the intensity profiles of the elastic and Raman scattering of an evaporating aqueous sodium nitrate droplet. We first describe Kerker's model and present some of the key results for elastic and Raman scattering from microspheres. From these equations, the relationships between resonances in elastic and Raman spectra can be understood. Experimental measurements of the elastically scattered light and Raman emissions from an evaporating sodium nitrate droplet will then be reported.

\section{Inelastic Scattering of Microspheres}

Kerker and co-workers ${ }^{12-15}$ developed a detailed model for inelastic scattering from microspheres. The microsphere is assumed to contain a Raman active molecule arbitrarily located at position $\mathbf{r}^{\prime}$ and that the boundary of the particle only affects the local exciting field inside the particle and the emitted radiation but not the molecular transitions. The field inside the particle consists of two parts, the internal field due to the incident excitation of frequency $\omega_{0}$ and a secondary field of a different frequency $\omega$ that represents the Raman emissions. The Raman emission is classically viewed as generated by a dipole representing the Raman active molecule located at $\mathbf{r}^{\prime}$, which undergoes forced oscillations at frequency $\omega$ induced by the local excitation field. The strength of the induced dipole $\mathbf{p}$ on which the Raman field depends is described as the product of an effective polarizability $\alpha$ and the local excitation field. The secondary field of frequency $\omega$ in the particle is the sum of the induced dipole field and an internal field due to the medium of the microsphere which is postulated to account for the effects of the boundary of the particle. Outside the particle, there are two outgoing fields: one of frequency $\omega$ due to Raman scattering from the particle and another of frequency $\omega_{0}$ from the elastic scattering. Here we present the key expressions for the elastic internal and external fields and inelastic external fields for Raman emissions. Using these equations, we examine the relationship of resonances of elastic and Raman scattering from a spherical particle. This analysis is important to the interpretation of resonance peaks in the Raman spectra reported later in this paper.

In the following equations, the media inside and outside the particle are labeled 1 and 2, respectively, with magnetic permeabilities $\mu_{1}$ and $\mu_{2}$, wavenumbers $k_{1}$ and $k_{2}$, and refractive indices $n_{1}$ and $n_{2}$. The particle radius is $a$. The local excitation electric field $\mathbf{E}(\mathbf{r})$ and magnetic field $\mathbf{B}(\mathbf{r})$ of frequency $\omega_{0}$ at location $\mathbf{r}$ inside the particle, which were also called the transmitted fields in Chew et al. ${ }^{15}$ may be written as a series of vector spherical harmonics:

$$
\begin{aligned}
& \mathbf{E}_{1}(\mathbf{r})=\sum_{l, m}\left\{\left(i c / n_{1}^{2} \omega_{0}\right) \gamma_{E}(l, m) \nabla x\left[j_{l}\left(k_{1} r\right) \mathbf{Y}_{l l m}(\hat{r})\right]\right. \\
& \left.\quad+\gamma_{M}(l, m) j_{l}\left(k_{1} r\right) \mathbf{Y}_{l l m}(\hat{r})\right\} \\
& \begin{aligned}
\mathbf{B}_{1}(\mathbf{r})=\sum_{l, m}\left\{\gamma_{E}(l, m) j_{l}\left(k_{1} r\right) \mathbf{Y}_{l l m}(\hat{r})\right. \\
\left.-\left(i c / \omega_{0}\right) \gamma_{M}(l, m) \nabla x\left[j_{l}\left(k_{1} r\right) \mathbf{Y}_{l l m}(\hat{r})\right]\right\}
\end{aligned}
\end{aligned}
$$

where $r=\|\mathbf{r}\|$ and $\hat{r}=\mathbf{r} / r$. The units and other notation are the same as in Jackson, ${ }^{16}$ except for the vector spherical harmonics which follow Edmonds. ${ }^{17}$ The internal field coefficients $\gamma_{E}(l, m)$ and $\gamma_{M}(l, m)$ are 


$$
\begin{aligned}
& \gamma_{E}(l, m)=\frac{\left(i n_{1}^{2} \mu_{1} / k_{2} a\right) \alpha_{E}(l, m)}{n_{1}^{2} \mu_{2} j_{l}\left(k_{1} a\right)\left[k_{2} a h_{l}^{(1)}\left(k_{2} a\right)\right]^{\prime}-n_{2}^{2} \mu_{1} h_{l}^{(1)}\left(k_{2} a\right)\left[k_{1} a j_{l}\left(k_{1} a\right)\right]^{\prime}}, \\
& \gamma_{M}(l, m)=\frac{-\left(i \mu_{1} / k_{2} a\right) \alpha_{M}(l, m)}{\mu_{2} h_{l}^{(1)}\left(k_{2} a\right)\left[k_{1} a j_{l}\left(k_{1} a\right)\right]^{\prime}-\mu_{1} j_{l}\left(k_{1} a\right)\left[k_{2} a h_{l}^{(1)}\left(k_{2} a\right)\right]^{\prime}},
\end{aligned}
$$

where $j_{l}$ and $h_{l}^{(1)}$ denote spherical Bessel functions of order $l$,

$$
\{x f(x)\}^{\prime} \equiv \frac{d}{d x}\{x f(x)\},
$$

and $\alpha_{E}(l, m)$ and $\alpha_{M}(l, m)$ are the incident field coefficients, functions of $l$ and $m$ only.

Similarly, the external elastically scattered field outside the particle can be described as

$$
\begin{aligned}
& \mathbf{E}_{\mathrm{sc}}(\mathbf{r})=\sum_{l, m}\left\{\left(i c / n_{2}^{2} \omega_{0}\right) \beta_{E}(l, m) \nabla x\left[h_{l}^{(1)}\left(k_{2} r\right) \mathbf{Y}_{l l m}(\hat{r})\right]\right. \\
& \left.+\beta_{M}(l, m) h_{l}^{(1)}\left(k_{2} r\right) \mathbf{Y}_{l l m}(\hat{r})\right\}, \\
& \mathbf{B}_{\mathrm{sc}}(\mathbf{r})=\sum_{l, m}\left\{\beta_{E}(l, m) h_{l}^{(1)}\left(k_{2} r\right) \mathbf{Y}_{l l m}(\hat{r})\right. \\
& \left.-\left(i c / \omega_{0}\right) \beta_{M}(l, m) \nabla x\left[h_{l}^{(1)}\left(k_{2} r\right) Y_{l l m}(\hat{r})\right]\right\},
\end{aligned}
$$

where the external elastically scattered field coefficients $\beta_{E}(l, m)$ and $\beta_{M}(l, m)$ are

$$
\begin{aligned}
& \beta_{E}(l, m) \\
& =\frac{\left\{n_{2}^{2} \mu_{1} j_{l}\left(k_{2} a\right)\left[k_{1} a j_{l}\left(k_{1} a\right)\right]^{\prime}-n_{1}^{2} \mu_{2} j_{l}\left(k_{1} a\right)\left[k_{2} a j_{l}\left(k_{2} a\right)\right]^{\prime} \alpha_{E}(l, m)\right.}{n_{1}^{2} \mu_{2} j_{l}\left(k_{1} a\right)\left[k_{2} a h_{l}^{(1)}\left(k_{2} a\right)\right]^{\prime}-n_{2}^{2} \mu_{1} h_{l}^{(1)}\left(k_{2} a\right)\left[k_{1} a j_{l}\left(k_{1} a\right)\right]^{\prime}}, \\
& \beta_{M}(l, m) \\
& \quad=\frac{\left\{\mu_{1} j_{l}\left(k_{1} a\right)\left[k_{2} a j_{l}\left(k_{2} a\right)\right]^{\prime}-\mu_{2} j_{l}\left(k_{2} a\right)\left[k_{1} a j_{l}\left(k_{1} a\right)\right]^{\prime}\right\} \alpha_{M}(l, m)}{\mu_{2} h_{l}^{(1)}\left(k_{2} a\right)\left[k_{1} a j_{l}\left(k_{1} a\right)\right]^{\prime}-\mu_{1} j_{l}\left(k_{1} a\right)\left[k_{2} a h_{l}^{(1)}\left(k_{2} a\right)\right]^{\prime}},
\end{aligned}
$$

Note that the $k_{1} a$ and $k_{2} a$ terms in the $\gamma$ and $\beta$ terms refer to light of frequency $\omega_{0}$, the incident light frequency. The poles of the $\gamma$ and $\beta$ terms determine the conditions for resonances. Since the coefficients $\alpha_{E}$ and $\beta_{E}, \alpha_{M}$ and $\beta_{M}$ have the same denominators, both the internal and external fields will exhibit resonance when $\omega_{0}$ is coincident with a resonance frequency of the particle. In a similar fashion, the Raman field at frequency $\omega$ outside the particle can also be written as

$$
\begin{aligned}
\mathbf{E}_{2}(\mathbf{r})=\sum_{l, m}\left\{\left(i c / n_{2}^{2} \omega\right) c_{E}(l, m) \nabla x\left[h_{l}^{(1)}\left(k_{2} r\right) \mathbf{Y}_{l l m}(\hat{r})\right]\right. & \\
& \left.+c_{M}(l, m) h_{l}^{(1)}\left(k_{2} r\right) Y_{l l m}(\hat{r})\right\}, \\
\mathbf{B}_{2}(\mathbf{r})=\sum_{l, m}\left\{c_{E}(l, m) h_{l}^{(1)}\left(k_{2} r\right) \mathbf{Y}_{l l m}(\hat{r})\right. & \\
& \left.-(i c / \omega) c_{M}(l, m) \nabla x\left[h_{l}^{(1)}\left(k_{2} r\right) \mathbf{Y}_{l l m}(\hat{r})\right]\right\}
\end{aligned}
$$

where the Raman outgoing field coefficients $c_{E}(l, m)$ and $c_{M}(l, m)$ are

$$
\begin{aligned}
& c_{E}(l, m) \\
& \quad=\frac{\left(i n_{2}^{2} \mu_{2} / k_{1} a\right) a_{E}(l, m)}{n_{1}^{2} \mu_{2} j_{l}\left(k_{1} a\right)\left[k_{2} a h_{l}^{(1)}\left(k_{2} a\right)\right]^{\prime}-n_{2}^{2} \mu_{1} h_{l}^{(1)}\left(k_{2} a\right)\left[k_{1} a j_{l}\left(k_{1} a\right)\right]^{\prime}},
\end{aligned}
$$

$$
\begin{aligned}
c_{M}(l, m) & \\
= & \frac{-\left(i \mu_{2} / k_{1} a\right) a_{M}(l, m)}{\mu_{2} h_{l}^{(1)}\left(k_{2} a\right)\left[k_{1} a j_{l}\left(k_{1} a\right)\right]^{\prime}-\mu_{1} j_{l}\left(k_{1} a\right)\left[k_{2} a h_{l}^{(1)}\left(k_{2} a\right)\right]^{\prime}},
\end{aligned}
$$

It should be noted that some of the field coefficients listed above are not identically the same as those in Chew et al. ${ }^{15}$ We have substituted the dielectric constant $\epsilon$, which was used in their original equations with $\mu$ and $n$, using

$$
n_{1}^{2}=\mu_{1} \epsilon_{1}, \quad n_{2}^{2}=\mu_{2} \epsilon_{2},
$$

so that the field coefficients can be compared more clearly.

The coefficients $a_{E}$ and $a_{M}$ in Eqs. (11) and (12), respectively, are the dipole field coefficients referring to a specific dipole representing a Raman active molecule localized at $\mathbf{r}^{\prime}$. However, they are proportional to the dipole moment $\mathbf{p}$ which can be described by the product of an effective polarizability $\alpha$ and the local transmitted field $\mathbf{E}_{1}(\mathbf{r})$ of frequency $\omega_{0}$. Hence $c_{E}$ and $c_{M}$ are both proportional to $\mathbf{E}_{1}\left(\mathbf{r}^{\prime}\right)$. If $\omega_{0}$ corresponds to a resonance frequency for $\mathbf{E}_{1}\left(\mathbf{r}^{\prime}\right)$, the Raman scattering field will also be at resonance, regardless of the frequency $\omega$ at which the Raman emissions occur. Therefore, one can expect that resonances in the entire Raman spectrum at all $\omega$ 's and in the elastic spectrum can occur simultaneously. More precisely, resonance in the elastic scattering is a necessary and sufficient condition for simultaneous resonances of Raman emissions at all frequencies.

Note that the $\gamma_{E}$ and $c_{E}$ (and similarly $\gamma_{M}$ and $c_{M}$ ) have the same form of denominator, although the argument in the latter refers to the Raman emission frequency $\omega$, while that of the former refers to the incident excitation frequency $\omega_{0}$. Since $\gamma_{E}$ and $c_{E}$ have the same form of denominator, one can see that resonance in the Raman scattering field can occur in a similar fashion as in elastic scattering. When $\omega$ corresponds to a resonant frequency, resonance occurs and the Raman emission at this particular frequency will be selectively enhanced. Therefore, one can expect resonances in a Raman spectrum to occur at selected wavelengths independent of each other as have been observed in broadband Raman spectra of droplets ${ }^{3,7,8}$ and glass microspheres. ${ }^{4-6}$

\section{Evaporation of an Aqueous $\mathrm{NaNO}_{3}$ Droplet Levitated in an Electrodynamic Balance}

A conventional bihyperboloidal electrodynamic blance was used to trap and levitate a droplet stationary for the Raman experiment. Details of the apparatus, which is illustrated in Fig. 1, have been reported elsewhere. ${ }^{18}$ A droplet of sodium nitrate solution was charged and introduced into the balance. After it was dried at vacuum, water vapor from a thermostated water reservoir was introduced, and the droplet was equilibrated to a constant size. Then, as the pressure in the balance was slowly reduced, the droplet evaporated and the elastically scattered light exhibited several resonance peaks during this size change. Both the 


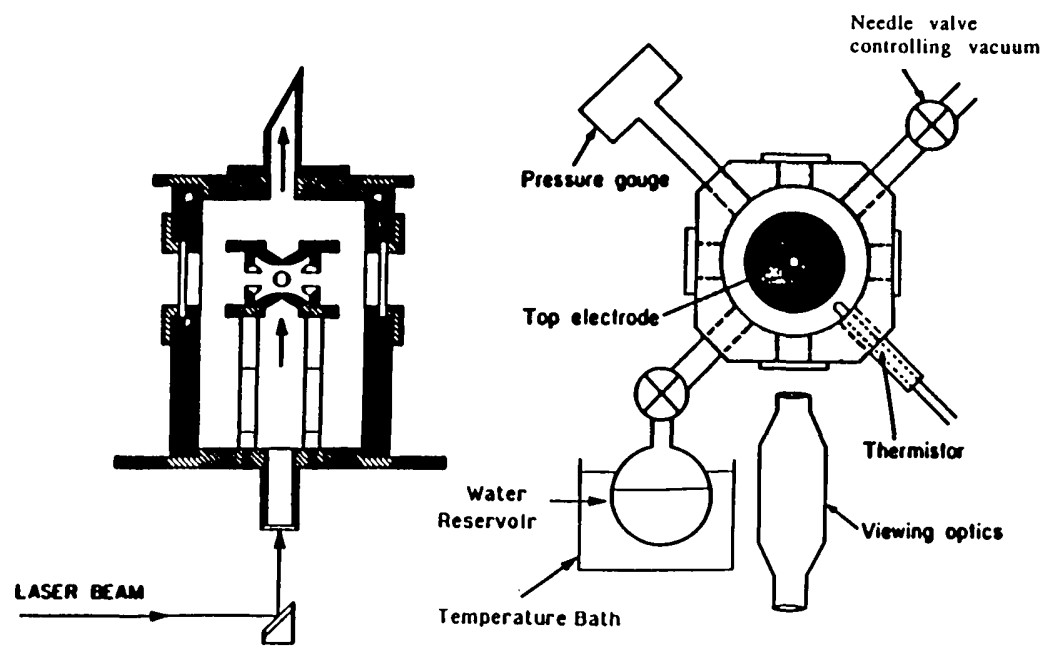

Fig. 1. Electrodynamic balance.

Raman spectra and elastically scattered light were recorded as a function of time.

To illustrate the two types of resonance described above, the solution studied should contain at least one Raman active component, preferably two, so that the scattering intensities for different Raman shifts can be compared. The Raman spectrum of a sodium nitrate solution exhibited a nitrate peak at $\sim 1040 \mathrm{~cm}^{-1}$ and a broad water peak at $3200-4000 \mathrm{~cm}^{-1}$. Another advantage of using a sodium nitrate droplet is that one can control the rate of evaporation easily. The rate of evacuation was manually adjusted using a needle valve so that appreciable change in the intensity of the elastically scattered light could be observed. The original size of the droplet was $\sim 20 \mu \mathrm{m}$ in diameter. The evaporation of water caused an $\sim 12 \%$ reduction in weight, i.e., a $4 \%$ reduction in particle size.

\section{Elastic and Raman Scattering Intensity Profiles of an Evaporating $\mathrm{NaNO}_{3}$ Droplet}

Figure 2 shows the apparatus used to measure the Raman scattering from single particles. A SpectraPhysics 2020 Ar-ion laser at $488.0 \mathrm{~nm}$ was used as the excitation source. It was operated at a nominal power of $0.5 \mathrm{~W}$. The laser beam was focused on the particle by a lens with a focal length of $125 \mathrm{~cm}$. The spectra of $90^{\circ}$ scattered light, containing the scattered laser light and the Raman nitrate and water peaks, were recorded by a Princeton Instrument IRY 700 optical multichannel analyzer (OMA). The OMA was operated at $20^{\circ} \mathrm{C}$ with the photocathode cooled to about $-25^{\circ} \mathrm{C}$ by flowing chilled methanol. It was attached to a SPEX $340 \mathrm{E}$ monochromator with a dispersion of $10 \mathrm{~nm} / \mathrm{mm}$ using a 300 -groove/mm grating, which has a spectral range sufficient to cover the laser scattered light and the Raman nitrate and water peaks. The OMA was controlled by a Princeton Instrument ST120 controller which was interfaced to an AT compatible computer. The measured Raman spectra were automatically stored in a hard disk via direct memory access.

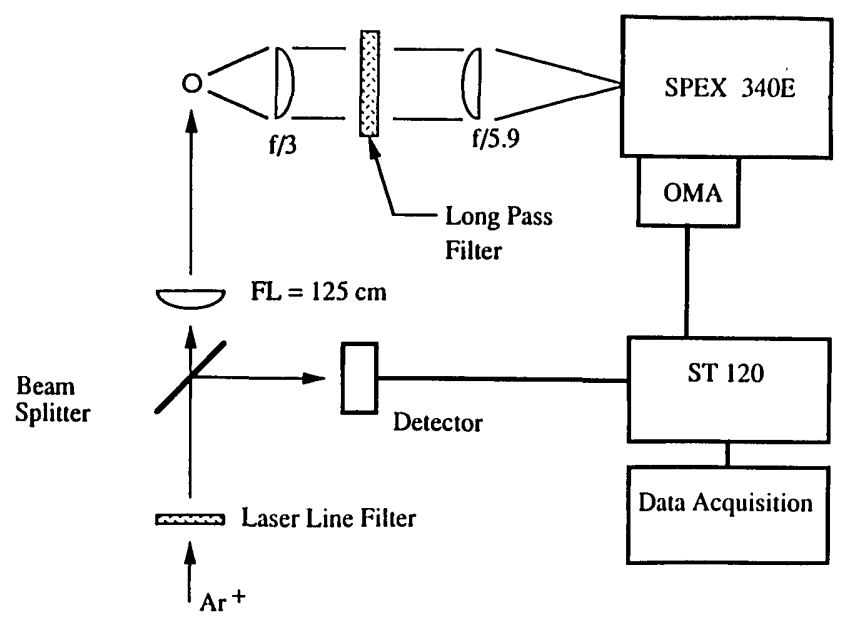

Fig. 2. Raman spectroscopy of single particles using an OMA.

Since the laser scattered light was orders of magnitude more intense than the Raman signal, a series of long pass filters was employed to attenuate the intensity of the elastically scattered light until the laser light and Raman signal fell into the same dynamic range of the OMA. By multiplexing the spectra using the OMA, the laser scattered light and Raman emissions from the particle were monitored simultaneously in real time as the droplet evaporated. Because the dielectric filter used was very sensitive to optical alignments, light scattered from the particle was collimated by a $f / 3$ lens before passing through the filters. After the filters, it was focused on the slit of the monochromator by a $f / 5.9$ lens, which matches the $f /$ No. of the monochromator. To shorten the integration time of each Raman measurement, the slit of the monochromator was adjusted to $200 \mu \mathrm{m}$. Beyond this value, the peak of the laser light only broadened without increasing its peak value. This slit width limits the spectral resolution to $14-19 \mathrm{~cm}^{-1}$. The integration time employed was $1.6 \mathrm{~s}$. 


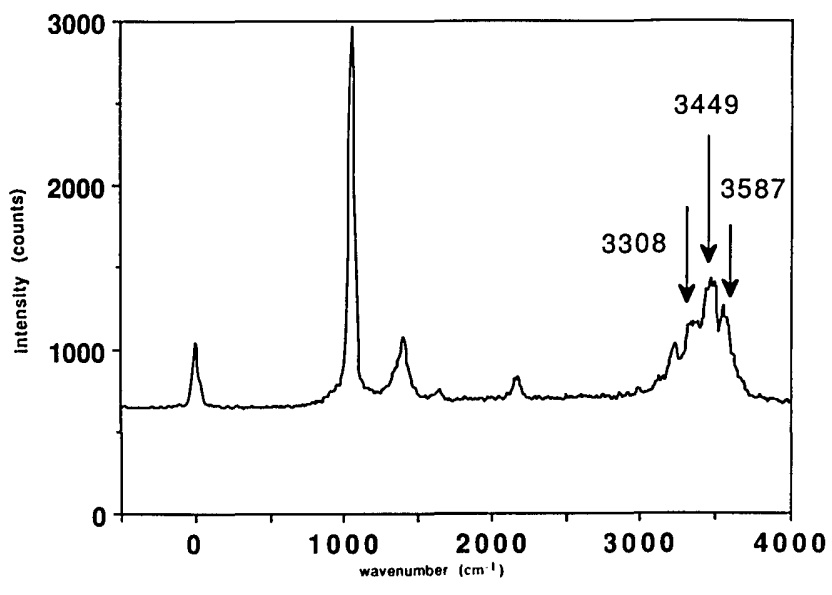

Fig. 3. Example of the Raman spectrum of an aqueous sodium nitrate droplet measured by an OMA.

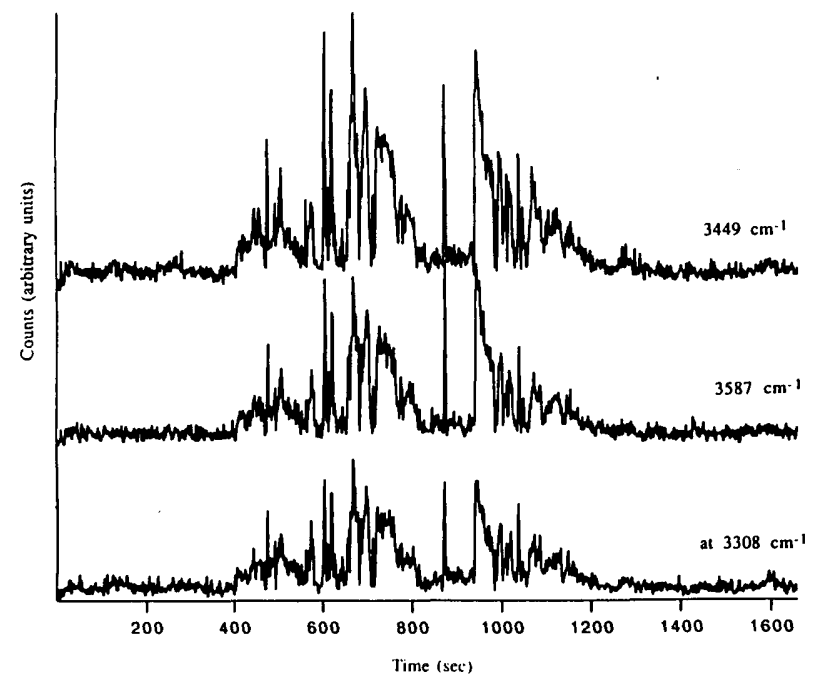

Fig. 4. Raman water intensity profiles at 3308,3349 , and 3587 $\mathrm{cm}^{-1}$.

Figure 3 shows a typical scattering spectrum of a sodium nitrate droplet measured by the OMA. The peak at $0 \mathrm{~cm}^{-1}$ is the elastically scattered Ar laser light, which has been attenuated by a factor of $10^{4}$. The peaks at 1040 and $1400 \mathrm{~cm}^{-1}$ are the nitrate peaks. The strong $1040-\mathrm{cm}^{-1}$ peak was taken as the representative of the nitrate emissions. The minor peak at $\sim 2100 \mathrm{~cm}^{-1}$ was due to room light at $546.07 \mathrm{~nm}$ leaking into the optical detection system. Since water is broadbanded from 3200 to $3800 \mathrm{~cm}^{-1}$, it is important to choose a representative wavenumber for the Raman emissions. Three intensity profiles, located at 3308 , 3449 , and $3587 \mathrm{~cm}^{-1}$, were examined. These correspond to the measured intensities at the half-maximum on the left, at the maximum, and at the halfmaximum on the right, as illustrated in Fig. 3, respectively. Figure 4 shows the time dependence of the intensities at these three wavenumbers in the broadband water peak. Although the water peak shown in Fig. 3 appears to exhibit resonances at vari-
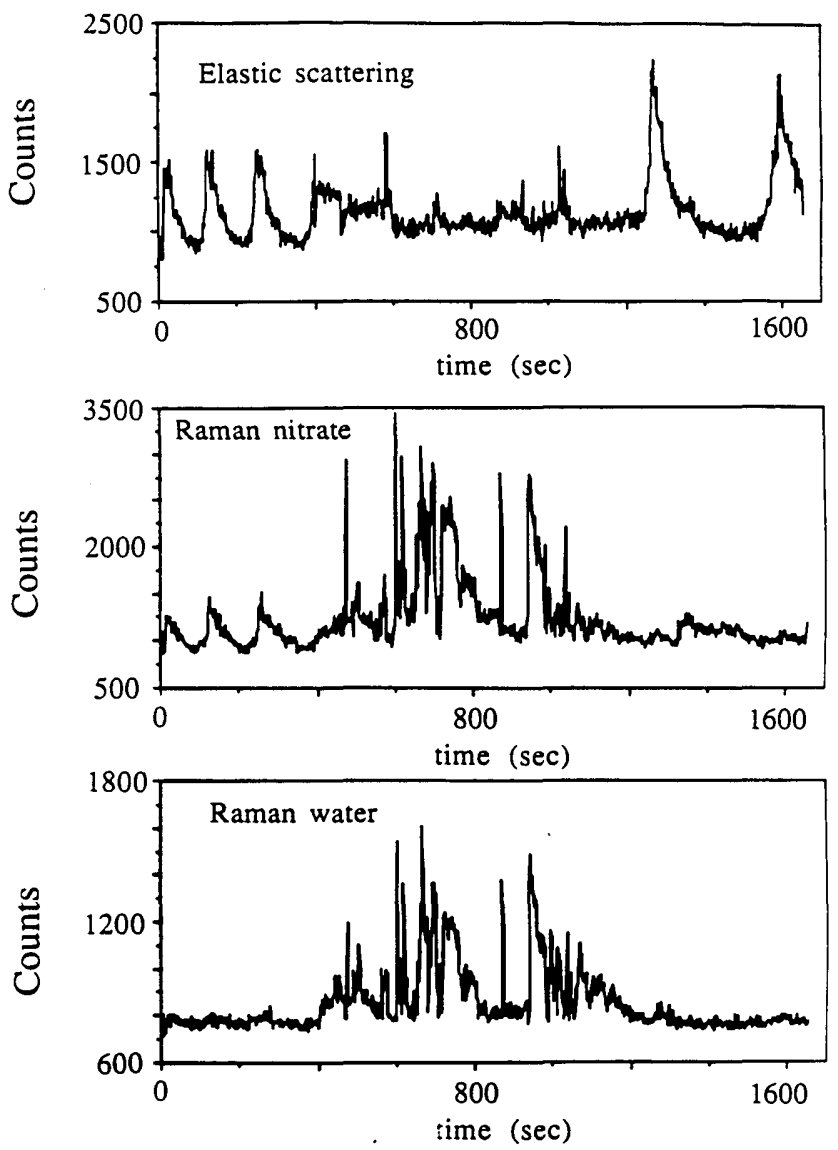

Fig. 5. Intensity profiles of the elastically scattered Ar line and Raman emissions of the nitrate and water peaks of an evaporating sodium nitrate droplet.

ous wavenumbers, it is clear from Fig. 4 that any one of the three wavenumbers can be used to represent the Raman water peak in the intensity profile.

To study the resonance structures in elastic and inelastic scattering of the evaporating droplet, the time variation of peak counts of the Ar laser line at 0 $\mathrm{cm}^{-1}$, the Raman nitrate peak at $1040 \mathrm{~cm}^{-1}$, and the Raman water peak at $3449 \mathrm{~cm}^{-1}$ are shown in Fig. 5 . Resonance structures in the Ar line, the Raman nitrate, and water emissions exhibit behaviors that can be grouped in three different time regions: (1) $0-400 \mathrm{~s}$; (2) $400-1200 \mathrm{~s}$; and (3) $1200-1650 \mathrm{~s}$. To facilitate the explanation of different observations in these three regions, we analyze the results of each region separately.

(1) $0-400 \mathrm{~s}$. There are three distinct major peaks in the elastic spectrum and the Raman nitrate spectrum as shown in Fig. 6. The water intensity profile also exhibits resonance structures at the same time albeit to a lesser extent. Since the elastically scattered light and the Raman emissions of both the nitrate and water exhibit resonances simultaneously, these resonances can be attributed to the resonance of the Ar laser line itself.

(2) 400-1200 s. The Raman intensity profiles of the nitrate and water peaks from 400 to $1200 \mathrm{~s}$ are 

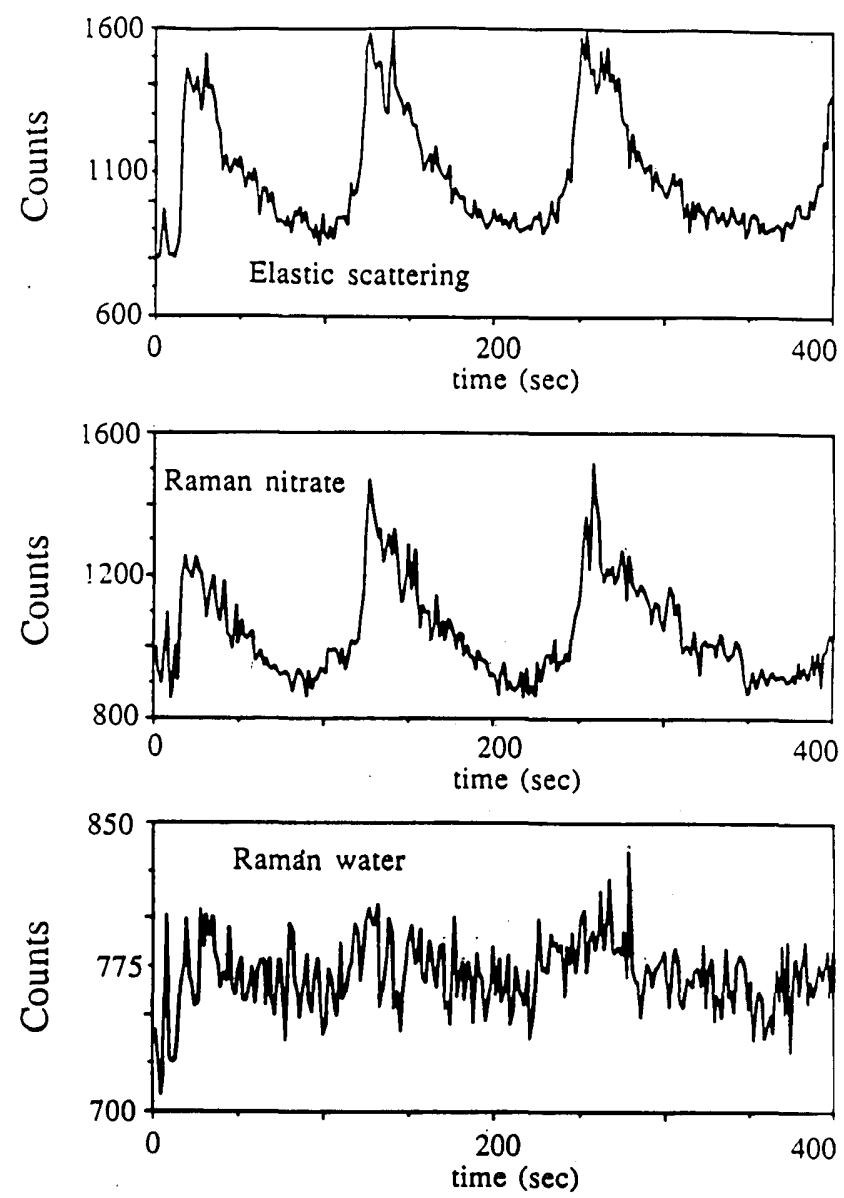

Fig. 6. Scattering intensity profiles from 0 to $400 \mathrm{~s}$.

shown in Fig. 7. One can clearly observe that the nitrate and water profiles have exactly the same structures. Also, the peaks here are much sharper than those three appearing in Fig. 6. The simultaneous appearance of resonances of the nitrate and water $\mathrm{Ra}$ man peak should be due to the internal resonance of the elastic scattered light. However, the resonance of the elastically scattered Ar laser light appeared at locations different from those of the Raman emissions. To illustrate this with a better resolution, Fig. 8 shows the elastically scattered Ar light intensity profile and the Raman nitrate intensity profile only between 400 and $800 \mathrm{~s}$. It is clear that resonances of the two intensity profiles occur at totally different locations, which seems to contradict the above criterion for the concurrent appearance of the Raman nitrate and water resonances. We explain this observation with the nature of low-order resonance peaks later.

(3) $1200-1650 \mathrm{~s}$. There are two distinct resonance peaks in the elastic spectrum, but the corresponding peaks in the Raman emissions are much weaker as depicted in Fig. 9. On the other hand, there are several small peaks in the nitrate spectrum occurring at $\sim 1350-1475 \mathrm{~s}$. They may be due to selective resonance of the nitrate peak when the nitrate wavelength corresponds to a resonant wavelength of the droplet at a particular size.
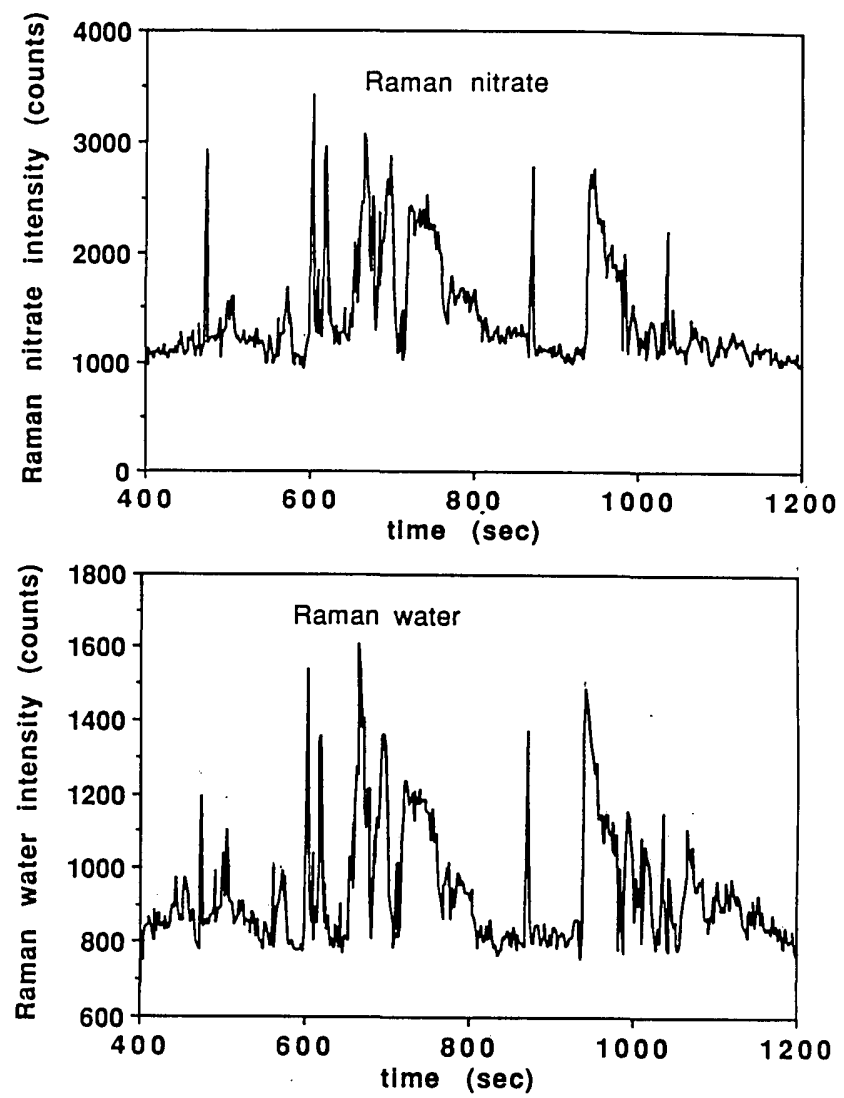

Fig. 7. Scattering intensity profiles of nitrate and water peaks from 400 to $1200 \mathrm{~s}$.

Efforts to use Raman spectroscopy to follow chemical reactions within aerosol particles depend on the relative intensities of different Raman lines and the elastically scattered light. It is, therefore, interesting to examine the Raman nitrate intensity and the Raman water intensity as a function of the Ar scattered intensity at various times. Figure 10(a) shows that the Raman nitrate vs Ar line plot exhibits a L shaped curve. Figure 10(b)-(d) show the contributions of data from regions (1) $0-400 \mathrm{~s}$, (2) 400-1200 s, and (3) $1200-1650 \mathrm{~s}$. The three temporal regions in the above analysis contribute to different regions of the $L$ shape plot. Figures 11(a)-(d) show similar plots for water intensity vs Ar line intensity. Figure 12 depicts the nitrate peak intensity as a function of the water peak intensity. In light of observations in Fig. 10 or 11 individually, it might be somewhat surprising to see that all the data points collapse into a rather linear zone as shown in Fig. 12. However, our analysis revealed that most of the resonance structures observed in this experiment were the result of resonance of the incident excitation light, which caused intensity enhancement of both the nitrate and water peaks to a similar extent. Therefore, a more or less linear relationship between the nitrate and water peak intensities can be expected. The location of this zone might give information regarding the concentration of the droplet (mole ratio of nitrate to water) albeit with a limited precision.

As emphasized in Fig. 7 and 8, we observed simulta- 

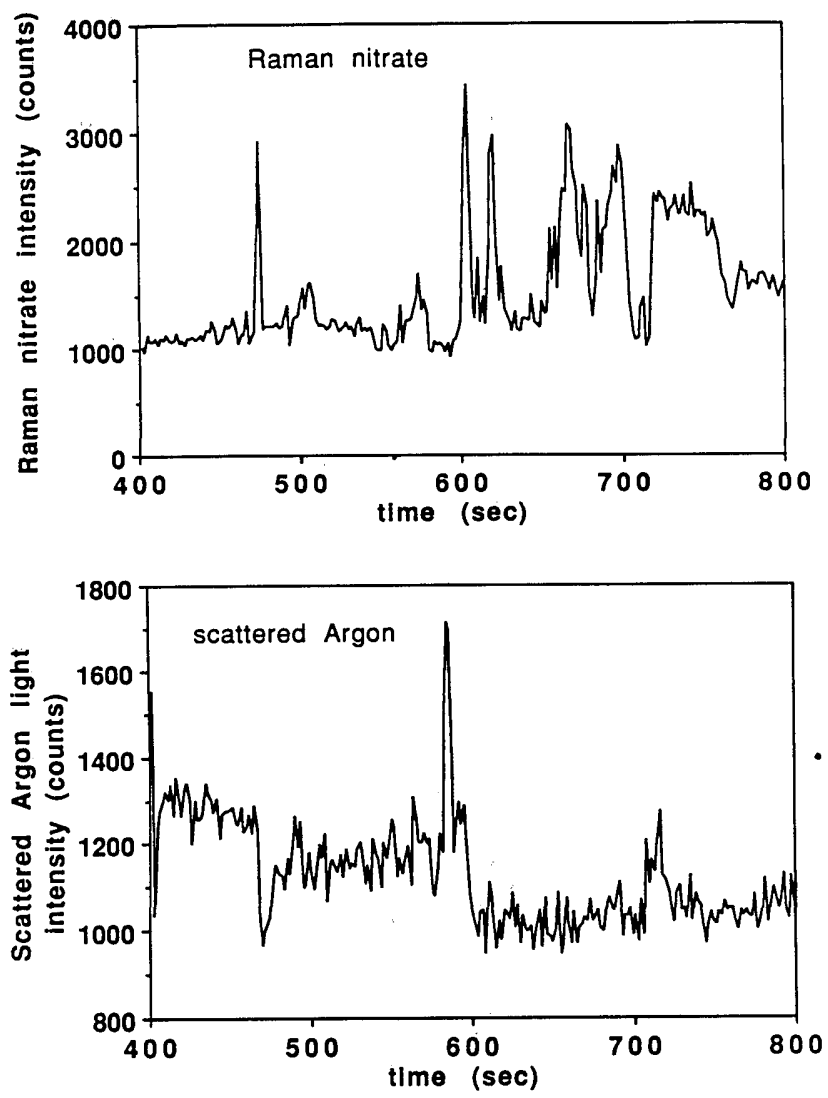

Fig. 8. Scattering intensity profiles of $\mathrm{Ar}$ line and nitrate peaks from 400 to $800 \mathrm{~s}$.
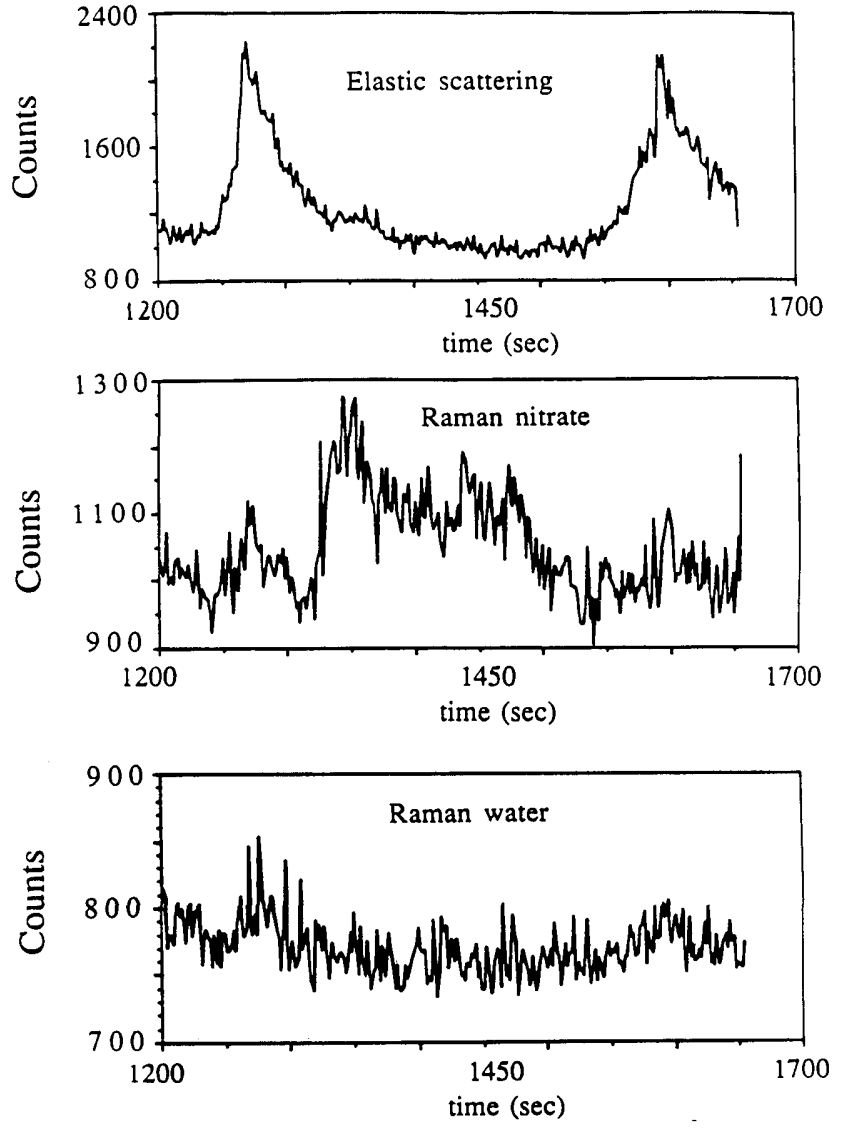

Fig. 9. Scattering intensity profiles from 1200 to $1650 \mathrm{~s}$.
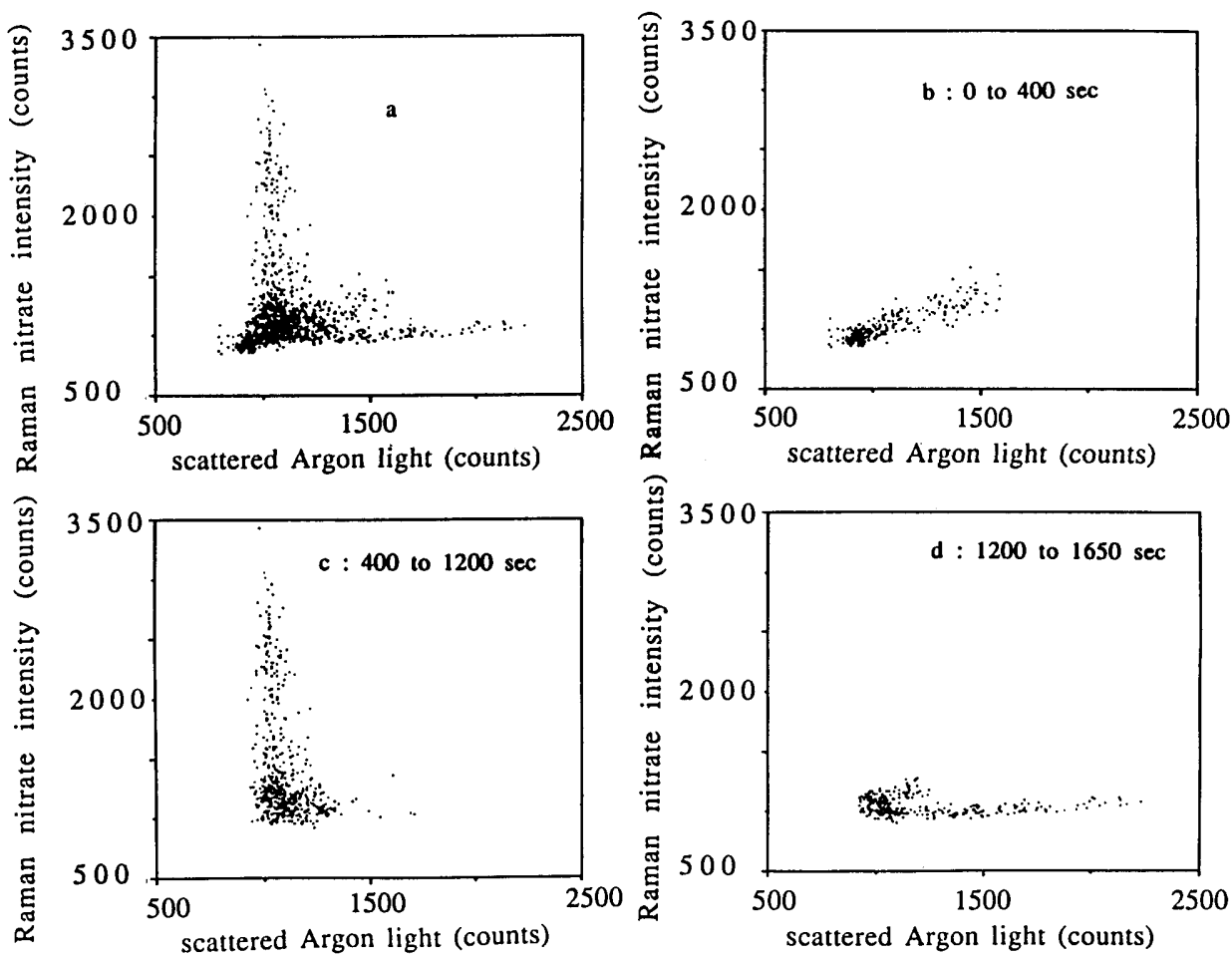

Fig. 10. Raman nitrate scattering intensity as a function of scattered Ar line intensity. 
Fig. 11. Raman water scattering intensity as a function of scattered Ar line intensity.
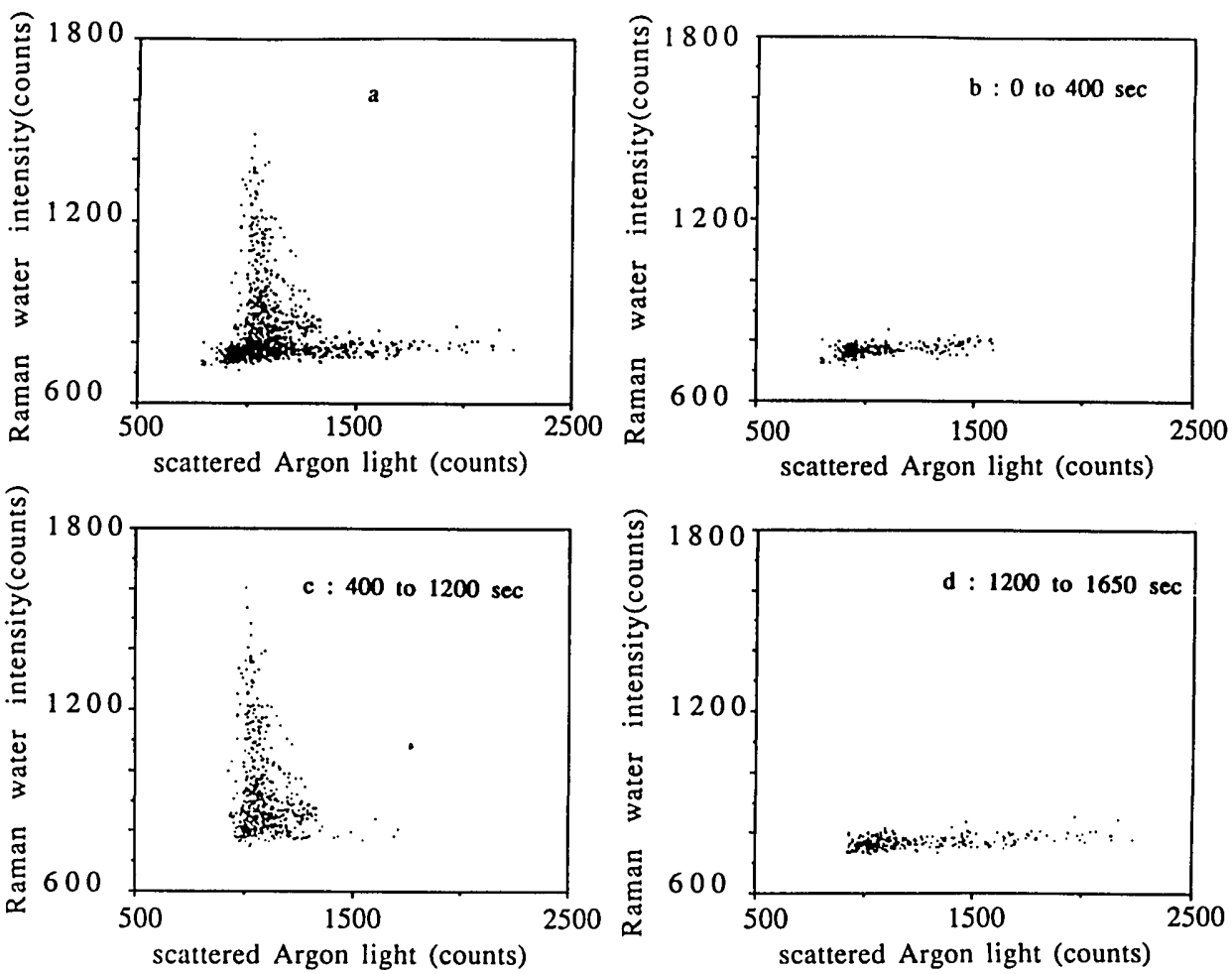

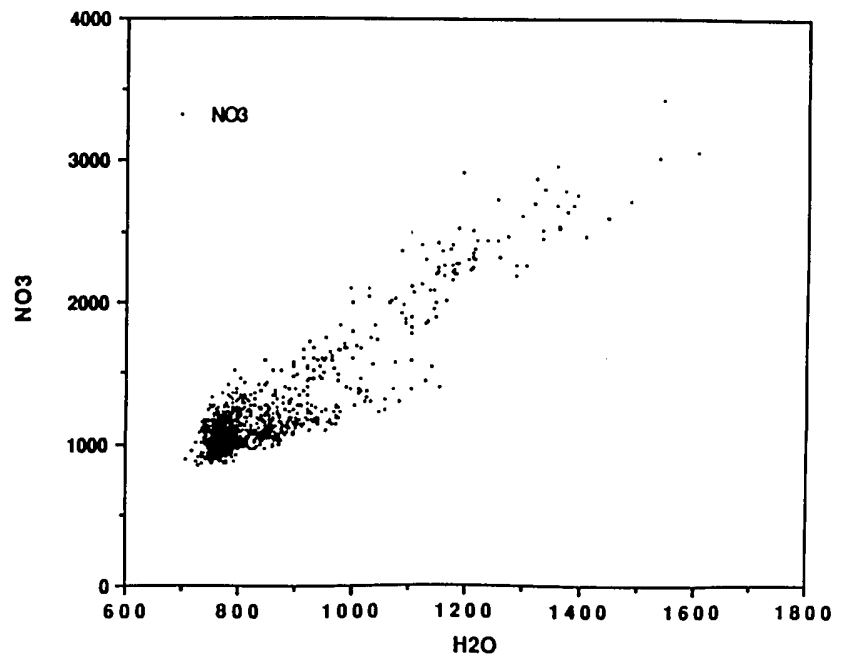

Fig. 12. Raman nitrate intensity as a function of Raman water scattering intensity.

neous resonance of the nitrate peak and the water peak without that occurring in the measured elastic intensity profile. This seems to contradict what we concluded earlier. Related phenomena have been reported by Qian and Chang. ${ }^{19}$ They found a much higher density of resonance peaks than normally observed in elastic spectra in multiple order Stokes emission in simulated Raman emissions from $\mathrm{CCl}_{4}$ microspheres. Hill and Benner 20,21 explained this observation by the characteristics of low-order resonance peaks in Mie scattering. We believe that our observation reported in this paper can also be explained in a similar manner.

\section{Low Order Resonance Structures in Mie Scattering}

Using Eqs. (7) and (8), when the denominator of the $\beta$ terms (or the $\gamma$ terms) becomes zero, the field will exhibit resonance structures. Each resonance peak can be assigned with a mode number and an order number. The mode number indicates the number of maxima between 0 and $180^{\circ}$ in the phase function, the angular distribution of energy of the mode. The order number indicates the number of maxima in the radial dependence of the mode. Mathematically a resonance mode number represents the collection of poles of the $\beta$ terms (or the $\gamma$ terms) of a particular $l$. At each $l$, there is a series of poles for the $\beta$ terms. Each pole of any particular $l$ is given an order number. The lower the order number, the sharper the resonance peak and also the more localized the energy in the particle is distributed near the surface. The sharpness of a resonance can be represented by $Q$, which is defined as the ratio of the resonant size parameter $X_{r}$ and the FWHM $\left(\Delta X_{1 / 2}\right)$ of a resonance peak. The lower the order of a resonance peak is, the smaller the $\Delta X_{1 / 2}$ is and the higher its $Q$ is.

Recall that the Raman scattering coefficients, $c_{M}$ and $c_{E}$, are proportional to the internal local excitation field strength, which is linear in $\gamma_{E}$ and $\gamma_{M}$, the internal field coefficients at $\omega_{0}$. On the other hand, the external elastically scattered field coefficients are $\beta_{E}$ and $\beta_{M}$. One should note that the signal registered by an OMA in an experiment was actually a time integrated intensity. In our evaporation experiment, the time in the intensity profiles can actually be replaced by the size parameter $X$. Therefore, for the case of a resonance peak, we are actually measuring the area of the 
peak which can be approximated by the product of peak intensity and $\Delta X_{1 / 2}$. Resonance structures with $Q>10^{5}$ are not generally observed in elastic spectra because the external field coefficients $\beta_{E}$ and $\beta_{M}$ are only an order of unity even at resonance. Hence the integrated area under a low order resonance peak in the elastic spectra approaches zero as $Q$ becomes very large (i.e., $\Delta X_{1 / 2}\left|\beta_{E}\right|^{2}$ and $\Delta X_{1 / 2}\left|\beta_{M}\right|^{2}$ go to zero). On the other hand, the internal field coefficients $\gamma_{E}$ and $\gamma_{M}$ can increase as $Q$ becomes large so that the product $\Delta X_{1 / 2}\left|\gamma_{E}\right|^{2}$ and $\Delta X_{1 / 2}\left|\gamma_{M}\right|^{2}$ can increase slowly to an order of about unity. This is basically due to the difference in the numerators of the $\beta$ and $\gamma$ terms. Physically most of the energy in a high $Q$ or high quality resonance is confined within a particle instead of leaking out as scattered light. Therefore, Raman enhancements due to low order internal resonance of the excitation field can occur without resonance in the elastic spectra being measured. This explains why the nitrate and water resonances occurred simultaneously while resonance in the elastic spectra was not measured. The sharpness of the peaks in Fig. 7 also supports this argument since they are much narrower than those observed in Fig. 6, where both the nitrate peak and elastically scattered light exhibit resonances.

\section{Conclusions}

By measuring simultaneously the elastically scattered light and Raman emissions from an evaporating aqueous sodium nitrate droplet as a function of time, one can study the interactions of Mie scattering with Raman emissions for spherical particles. We found that resonances of the elastically scattered light, $\mathrm{Ra}$ man nitrate peak, and Raman water peak can occur in a concerted mode and can also occur independent of each other. These observations can be explained by Kerker's ${ }^{13-15}$ model of inelastic scattering from microspheres and the behavior of low order resonance structures in Mie scattering. This type of measurement can be potentially used to test the validity of existing models of Raman emissions from microspheres. One application of this type of data would be quantitative determination of the chemical compositions of spherical particles. One could use the elastic spectra to obtain the refractive index and particle size of the droplet and then use accurate models of Raman emissions from spherical particles with the known particle size and refractive index to determine the chemical composition of the particle.

This research was supported by the Caltech Consortium in Chemistry and Chemical Engineering; founding members: E.I. du Pont de Nemours and Co., Inc., Eastman Kodak Co., Minnesota Mining and Manufacturing Co., and Shell Development Co.

\section{References}

1. S. Arnold, "Spectroscopy of Single Levitated Micron Sized Particles," in Optical Effects Associated with Small Particles, P. W. Barber and R. K. Chang, Ed. (World Scientific, Singapore, 1988).

2. G. J. Rosasco, E. S. Etz, and W. A. Cassatt, "The Analysis of Discrete Fine Particle by Raman Spectroscopy," Appl. Spectrosc. 29, 396-404 (1975).

3. R. E. Preston, T. R. Lettieri, and H. G. Semerjian, "Characterization of Single Levitated Droplets by Raman Spectroscopy," Langmuir 1, 365-367 (1985).

4. R. Thurn and W. Kiefer, "Observations of Structural Resonances in the Raman Spectra of Optically Levitated Dielectric Microspheres," J. Raman Spectrosc. 15, 411-413 (1984).

5. R. Thurn and W. Kiefer, "Raman-Microsampling Technique Applying Optical Levitation by Radiation Pressure," Appl. Spectrosc. 38, 78-83 (1984).

6. R. Thurn and W. Kiefer, "Structural Resonances Observed in the Raman Spectra of Optically Levitated Liquid Droplets," Appl. Opt. 24, 1515-1519 (1985).

7. K. H. Fung and I. N. Tang, "Raman Scattering from Single Solution Droplets," Appl. Opt. 27, 206-208 (1988).

8. K. H. Fung and I. N. Tang, "Raman Spectra of Singly Suspended Supersaturated Ammonium Bisulfate Droplets," Chem. Phys. Lett. 147, 509-513 (1988).

9. K. H. Fung and I. N. Tang, "Composition Analysis of Suspended Aerosol Particles by Raman Spectroscopy: Sulfates and Nitrates," J. Colloid and Interface Sci. 130, 219-224 (1989).

10. I. N. Tang and K. H. Fung, "Characterization of Inorganic Salt Particles by Raman Spectroscopy," J. Aerosol Sci. 20, 609-617 (1989).

11. R. E. Benner, P. W. Barber, J. F. Owen, and R. K. Chang, "Observation of Structure Resonances in the Fluorescence Spectra from Microspheres," Phys. Rev. Lett. 44, 475-478 (1980).

12. M. Kerker and S. D. Druger, "Raman and Fluorescent Scattering by Molecules Embedded in Spheres with Radii up to Several Multiples of the Wavelength," Appl. Opt. 18, 1172-1179 (1979).

13. M. Kerker, "Lorenz-Mie Scattering by Spheres: Some Newly Recognized Phenomena," Aerosol. Sci. Technol. 1, 275-291 (1982).

14. P. J. McNulty, H. W. Chew, and M. Kerker, "Inelastic Light Scattering," in Aerosol Microphysics I: Particle Interaction, W. H. Marlow, Ed. (Springer-Verlag, Berlin, 1980).

15. H. Chew, P. J. McNulty, and M. Kerker, "Model for Raman and Fluorescent Scattering by Molecules Embedded in Small Particles," Phys. Rev. A 13, 396-404 (1976).

16. J. D. Jackson, Classical Electrodynamics (Wiley, New York, 1962).

17. A. R. Edmonds, Angular Momentum in Quantum Mechanics (Princeton U.P., Princeton, NJ, 1957).

18. G. Sageev, R. C. Flagan, J. H. Seinfeld, and S. Arnold, "Condensation Rate of Water on Aqueous Droplets in the Transition Regime," J. Colloid. Interface Sci. 113, 421-429 (1986).

19. S.-X. Qian and R. K. Chang, "Multi-Order Stokes Emissions from Micrometer Sized Droplets," Phys. Rev. Lett. 56, 926 (1986).

20. S. C. Hill and R. E. Benner, "Morphology-Dependent Resonances Associated with Stimulated Processes in Microspheres," J. Opt. Soc. Am. B 3, 1509-1514 (1986).

21. S. C. Hill and R. E. Benner, "Morphology-Dependent Resonances," in Optical Effects Associated with Small Particles, P. W. Barber and R. K. Chang, Ed. (World Scientific, Singapore, 1988). 